\title{
Differential radiosensitivity of hypothalamo-pituitary function in the young adult rat
}

\author{
I C A F Robinson, K M Fairhall, J H Hendry ${ }^{1}$ and S M Shalet ${ }^{2}$
}

Division of Molecular Neuroendocrinology, National Institute for Medical Research, Mill Hill, London, UK

${ }^{1}$ Experimental Radiation Oncology Group, Paterson Institute for Cancer Research, Manchester, UK

${ }^{2}$ Department of Medicine and Endocrinology, Christie Hospital, Manchester, UK

(Requests for offprints should be addressed to I C A F Robinson, Division of Molecular Neuroendocrinology, National Institute for Medical Research, Mill Hill, London NW7 1AA, UK; Email: irobins@nimr.mrc.ac.uk)

\begin{abstract}
Cranial irradiation in children and adults often results in irreversible hypopituitarism. The earliest and most common endocrine abnormality is GH deficiency, often followed by other pituitary hormone deficits. We investigated whether a similar pattern of progressive hypopituitarism could be reproduced in an animal model. Different doses of cranial irradiation were delivered to the hypothalamo-pituitary region of normal adult male rats, and the effects on their subsequent growth, pituitary weight and hormone contents were studied. Animals received cranial irradiation with $300 \mathrm{kV}$ X-rays at doses of $0,20,22$ or $24 \mathrm{~Gy}$ ( $n=15$ per group) and five animals from each group were killed at 8,14 or 20 weeks after irradiation. Their anterior pituitary glands were weighed and assayed for $\mathrm{GH}, \mathrm{LH}$, TSH, ACTH and prolactin (PRL) content. All three doses of irradiation reduced body weight compared with that in non-irradiated controls and
\end{abstract}

compromised growth between 8 and 20 weeks. Pituitary weight increased between 8 and 20 weeks in control rats, whereas it decreased significantly in the irradiated animals. Irradiation induced time- and dose-dependent changes in pituitary hormone contents. GH and PRL were most sensitive and decreased by more than $90 \%$ after irradiation; TSH contents were unaffected 8 weeks after the lowest dose of irradiation, but were reduced at 14 and 20 weeks. $\mathrm{LH}$ and ACTH were the slowest to be affected, and only at the greater doses of radiation. Thus progressive multiple pituitary endocrine deficits can be induced differentially in rats by increasing doses of cranial irradiation. This model should prove useful for defining the sites and mechanisms by which cranial irradiation induces neuroendocrine dysfunction.

Journal of Endocrinology (2001) 169, 519-526

\section{Introduction}

Cranial irradiation is a relatively common cause of irreversible hypopituitarism in both children and adults. It has been observed in patients who have been given radiotherapy for nasopharyngeal carcinoma (Samaan et al. 1982, Lam et al. 1991), for tumours of the pituitary gland or nearby structures (Littley et al. 1989a,b), and for primary brain tumours (Harrop et al. 1976, Richards et al. 1976). It is also common in children who have been given prophylactic cranial irradiation for acute lymphoblastic leukaemia (Shalet \& Brennan 1998) or total-body irradiation for a variety of tumours and other diseases.

Hypopituitarism induced by cranial irradiation shows some characteristic features. The endocrine deficits can vary widely in onset and may be delayed months or years after irradiation but, once detected, the severity of pituitary dysfunction increases with time and is irreversible. The endocrine deficits can range from mild isolated growth hormone $(\mathrm{GH})$ deficiency to severe pan-hypopituitarism, though GH is commonly the first anterior pituitary hormone to be affected (Littley et al. 1989a). The findings of studies in adults who received external radiation therapy for pituitary adenomas or nasopharyngeal carcinomas suggest that deficiency of either gonadotrophin or adrenocorticotrophic hormone (ACTH) occurs later than that of $\mathrm{GH}$, and that thyroidstimulating hormone (TSH) is the least likely to be adversely affected (Littley et al. 1989a, Lam et al. 1991). In such patients, the relationship between dose and prevalence of pituitary hormone deficits is different from that seen in patients receiving direct intra-pituitary irradiation from yttrium-90 implants (Jadresic et al. 1987), and is believed to reflect hypothalamic damage from external irradiation that reduces the output of hypophysiotrophic factors required to maintain a normal pituitary gland.

The total dose of radiation delivered to the hypothalamo-pituitary region is a major determinant of the speed of onset, incidence and severity of anterior pituitary hormone deficiencies. The greater the radiation 
dose, the more likely it is that the patient will develop pan-hypopituitarism and the earlier the deficiencies will occur; the severity of the hormone deficiency is also a function of the period of time elapsed since irradiation (Littley et al. 1989b).

It is unlikely that much more can be learned about the mechanisms of irradiation-induced endocrine deficits from studies in humans. Apart from ethical considerations, it is difficult to pinpoint the sites and mechanisms of irradiation-induced neuroendocrine damage by means of measurements of hormones in blood. We therefore sought to develop an animal model in which a similar constellation of progressive multiple pituitary endocrine deficits might be induced in a predictable fashion by cranial irradiation, so that more direct measures of pituitary or hypothalamic cellular function could be made.

The animal species best characterized for such neuroendocrine studies is the rat, and previous work has shown that irradiation in neonatal or weanling rats will attenuate their growth and reduce GH secretion (Mosier et al. 1985, 1986), and will affect their rate of sexual maturation (Roth et al. 2000). However, it is not known whether irradiation in adult rats will induce progressive multiple pituitary hormone deficits in a dose- and time-dependent fashion analogous to those seen in humans. We have developed a simple procedure for delivering different doses of cranial irradiation to young adult normal rats, and have characterized directly the differential onset and severity of multiple pituitary hormone deficits that follow such treatment.

\section{Materials and Methods}

\section{Irradiation}

All experimental procedures were performed according to the Animals (Scientific Procedures) Act of Parliament, UK 1986. Normal young adult male rats $(n=60)$ of the NIMR:AS strain (8 weeks of age, weighing $150-190 \mathrm{~g}$ at the start of treatment) were lightly anaesthetized (i.p. ketamine/xylazine), placed in a positioning harness and irradiated using $300 \mathrm{kVp}$ X-rays (Guo et al. 1997) using a vertical beam through the head to a $1.5 \mathrm{~cm}$ diameter field that included the hypothalamo-pituitary region. The remainder of the brain and body were shielded by lead. Animals were randomly divided into four groups of 15 that received a single dose of $0,20,22$ or 24 Gy. At 8, 14 and 20 weeks after irradiation, five animals from each group were killed and their anterior pituitary glands excised, weighed and frozen for subsequent hormone assays.

\section{Hormone assays}

The pituitaries were thawed and homogenized in $1 \mathrm{ml}$ PBS, centrifuged at $10000 \mathrm{~g}$ for $2 \mathrm{~min}$, and the supernatants subjected to radioimmunoassays for $\mathrm{GH}$, prolactin (PRL), TSH, luteinizing hormone (LH) and ACTH using reagents obtained from NIDDK. The results are expressed as $\mu \mathrm{g}$ per pituitary, in terms of the appropriate reference standards: GH-RP2; PRL-RP3; TSH-RP3; LH-RP3; ACTH-RP1

\section{Statistical analysis}

Unless otherwise stated, data are shown as means \pm s.E.M., and differences between groups were assessed by ANOVA followed by Dunnett's test for multiple comparisons, with $P<0.05$ considered significant. Statistical analyses in Figs 1-3 were performed on raw data. However, because the individual hormones are present in very different amounts, to compare the sensitivity, onset and rate of decline between the different hormones, relative decay curves were constructed by expressing the data for each hormone as a percentage of the mean content of the same hormone measured in the non-irradiated controls at the corresponding time point, normalized to $100 \%$ and plotted relative to this (Fig. 4). In this case, the data were log-transformed and between-hormone comparisons at the same time point and irradiation dose were assessed by Student-Newmann-Keuls test.

\section{Results}

All three doses of irradiation resulted in a reduced body weight gain compared with that in non-irradiated controls, and this was already evident at 8 weeks after irradiation (Fig. 1a). Furthermore, over the following 12 weeks, the increase in body weight seen in the control rats was virtually abolished in the irradiated animals (Fig. 1a), though they were able to sustain a slight increase in body weight gain throughout this period. Apart from this growth deficit, the animals were in good general health.

More pronounced effects of irradiation were seen on pituitary size. Non-irradiated control rats showed an increase in pituitary weight over this period, whereas pituitary weight decreased significantly with time in all irradiated groups (Fig. 1b). This was not simply a consequence of reduced growth rate, as body weights were maintained over the same period; therefore, irradiation induced an absolute loss of pituitary mass. This was already evident 8 weeks after irradiation at $24 \mathrm{~Gy}$, and became apparent by 14 weeks in the animals receiving the lower doses. By 20 weeks after irradiation, all three groups had pituitaries less than half the weight of those of the corresponding non-irradiated animals (Fig. 1b).

Pituitary hormone contents for all 60 animals were measured; the data are presented in Figs 2 and 3. Changes in $\mathrm{GH}$ were dramatic (Fig. 2). Whereas control animals showed time-related increases in GH contents that paralleled the increase in pituitary weight over this time course, irradiated animals showed a marked decrease in pituitary GH that was dose- and time-dependent (Fig. 2). The dose effect was seen most clearly after 8 weeks, when 
(a)

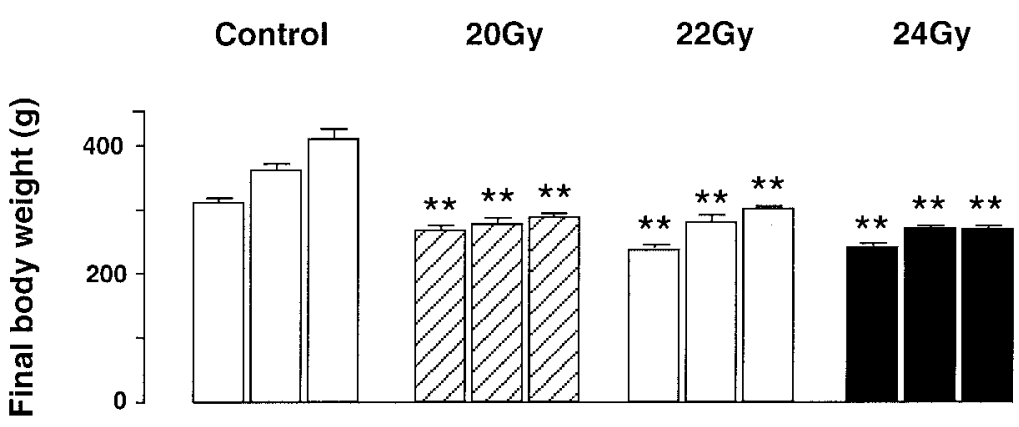

(b)

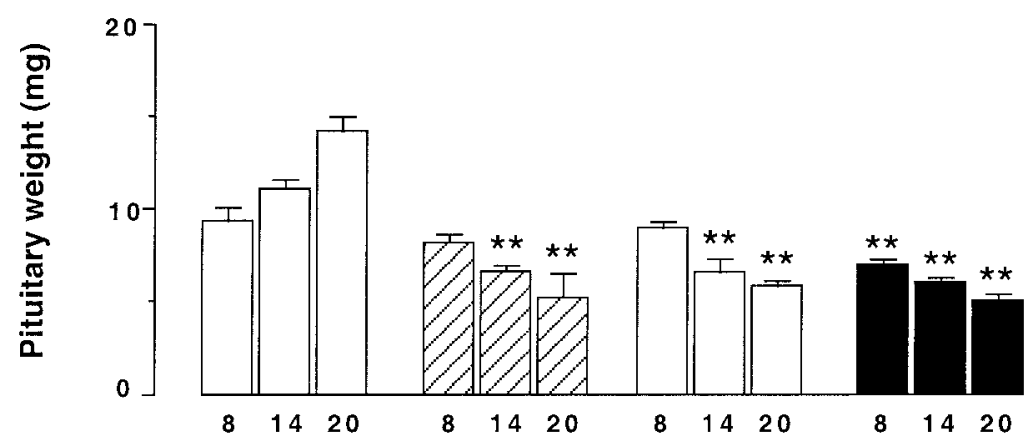

\section{Time post irradiation (weeks)}

Figure 1 The effects of cranial irradiation on (a) body weight and (b) anterior pituitary gland weight in rats. Rats ( $n=15$ per group) received 0 Gy (Control group, open bars), 20 Gy (hatched bars), 22 Gy (shaded bars) or 24 Gy irradiation (solid bars) and five animals from each group were killed 8, 14 or 20 weeks later. Body weight and anterior pituitary weights were recorded and are shown as means + S.E.M. ${ }^{* *} P<0 \cdot 01$ compared with the corresponding time point in controls (Dunnett's test).

the $\mathrm{GH}$ contents in the $20 \mathrm{~Gy}$ and $24 \mathrm{~Gy}$ animals were $<60 \%$ and $<15 \%$ of those in the control rats respectively. At each irradiation dose, the GH contents decreased progressively with time. After 20 weeks, pituitary GH contents in the group irradiated to the greatest dose (24 Gy) had decreased to less than $5 \%$ of those in non-irradiated animals at the same age.

The other pituitary hormones were also affected by irradiation but to different degrees (Fig. 3). The effects on PRL were very similar to those seen for GH, with more than $90 \%$ loss of pituitary PRL contents 20 weeks after irradiation to 24 Gy (Fig. 3). TSH contents also showed both time- and dose-dependent reductions, but the magnitude of the effects were less severe than for $\mathrm{GH}$ or PRL. After $20 \mathrm{~Gy}$ irradiation, there was no change in TSH at 8 weeks but there was a progressive reduction over the following 12 weeks. More pronounced effects were seen with the greater doses of irradiation; by 20 weeks after 24 Gy, TSH contents had decreased to around 20\% of those in the corresponding non-irradiated animals (Fig. 3).
It took longer for effects of irradiation on LH contents to become manifest. There were no significant effects observed in any group after 8 weeks. However, moderate, time-dependent reductions in pituitary LH content became apparent thereafter, most clearly in the rats that received the greatest dose of irradiation (Fig. 3).

ACTH contents were least affected by irradiation. The increase in ACTH that occurred with time in the control animals was reversed in all the irradiated groups, but the absolute deficits induced by irradiation were mild (Fig. 3). The group irradiated to the greatest dose (24 Gy) still maintained around $40 \%$ of the ACTH content of the control group after 20 weeks, and this decrease in ACTH content was not much greater than could be accounted for by the reduction in overall pituitary weight between these groups.

Relative decay curves for all the hormone contents were constructed by expressing the data for each hormone as a percentage of that measured at the corresponding timepoint in non-irradiated rats, each normalized to $100 \%$ (see Methods); the results are illustrated in Fig. 4. This clearly 


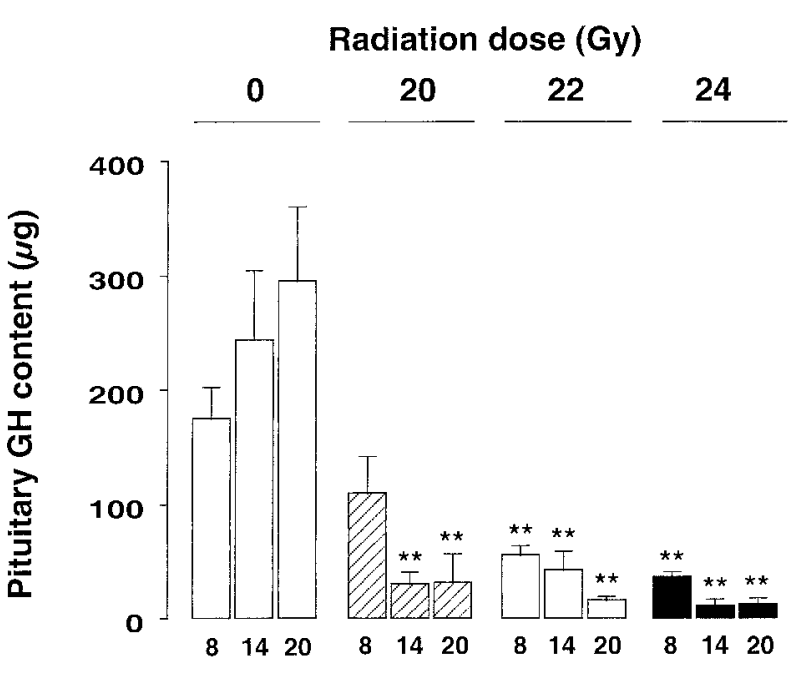

Time post irradiation (weeks)

Figure 2 Cranial irradiation reduces pituitary $\mathrm{GH}$ content in a dose- and time-dependent manner. The groups are the same as in Fig. 1 and received 0 Gy (Control group, open bars), 20 Gy (hatched bars), 22 Gy (shaded bars) or 24 Gy irradiation (solid bars). Pituitary GH contents were measured by RIA in rats killed 8, 14 or 20 weeks after cranial irradiation, and expressed as $\mu \mathrm{g} /$ pituitary. Data are means + S.E.M. ${ }^{* *} P<0 \cdot 01$ compared with the corresponding time point in controls (Dunnett's test).

shows the parallel early and profound effects of irradiation on GH and PRL and the differences in radiosensitivity, onset, rate of progression and severity for the other hormones measured.

\section{Discussion}

In a series of classic studies, Mosier and colleagues showed that cranial irradiation delivered to neonatal rats stunted their growth and compromised GH secretion (Mosier \& Jansons 1968, 1971, Mosier et al. 1985, 1986, Mosier 1988). Cranial irradiation in infant or weanling rats has also been investigated (Overmier et al. 1979, Schunior et al. 1990). In neonatal rats, the hypothalamo-pituitary structures are still forming and developing functional connections. We were interested in developing a similar irradiation model in older, post-pubertal rats and to look for differences in the radiosensitivity, onset, progression and severity of the damage between the different pituitary hormone axes in the same animals.

The choice of dose and field of irradiation was guided by earlier experiments that had shown that a $20 \mathrm{~Gy}$ dose delivered to a $1.0 \mathrm{~cm}$ diameter field had a significant but transient inhibitory effect on growth (Guo et al. 1997). We therefore chose $20 \mathrm{~Gy}$ as a baseline dose, and tested 22 and $24 \mathrm{~Gy}$ as higher doses. The field was also extended to $1.5 \mathrm{~cm}$ in diameter to ensure inclusion of the entire hypothalamus and pituitary region. However, as many other central nervous system structures were in the vertical beam path, we cannot assume that all the endocrine effects observed were solely due to hypothalamic or pituitary damage. Nevertheless, the rats tolerated all three doses well, and continued to show some small body weight gains, though all remained significantly smaller than the non-irradiated animals. Lower doses of cranial irradiation in much younger rats causes a permanent impairment in weight gain and bone growth, with a positive relationship between radiation dose and growth impairment (Overmier et al. 1979, Mosier et al. 1985, Schunior et al. 1990, Roth et al. 2000).

One important difference from clinical practice was our use of a single dose instead of a fractionated regime, because our objective was to establish a dose-response relationship in a simple procedure, readily applicable to several groups of animals. Roth et al. (2000) found that two doses of 9 Gy, delivered at 21 and 23 days to female rats, also caused marked growth retardation at 3 months of age, and fractionating the dose may well affect different cell populations to differing degrees (Thames \& Hendry 1987).

Normal young adult rats continue to show GHdependent growth, so biologically significant reductions in GH output should be reflected in a reduced rate of growth. In fact, all three doses of irradiation had similar effects on weight gain and pituitary weights, probably reflecting the fact that the GH and PRL systems were the first and most profoundly affected, with near maximal effects on GH after 14 weeks with all doses of irradiation. Although the changes in body weight could largely be explained by the time-course and severity of GH deficiency, they could also reflect damage to other hypothalamic centres (for example, those regulating food intake or energy expenditure), as these will also have been included within the irradiation field. It has also been shown that cranial irradiation in neonatal rats compromises their later food efficiency (Mosier et al. 1993). Clearly, it would be interesting to extend our studies to include measures of energy intake, expenditure, and body composition after cranial irradiation at these doses in young adult rats.

The observation that pituitary weight reductions were similar with all irradiation doses also could be explained by the early and relatively severe effects on the GH/PRL axes. The cell types synthesizing these hormones comprise about $50 \%$ of the total endocrine cell mass of the rat anterior pituitary, so an early loss of these cells would have a large effect on pituitary weight, even if the other pituitary cell types were relatively unaffected at this time.

There are surprisingly few data on the effects of irradiation on pituitary hormone contents. An early study found little effect of irradiation on pituitary hormone contents, but this was relatively insensitive and involved bioassays of pituitary extracts from irradiated animals in recipient hypophysectomized rats (Mosier \& Jansons 

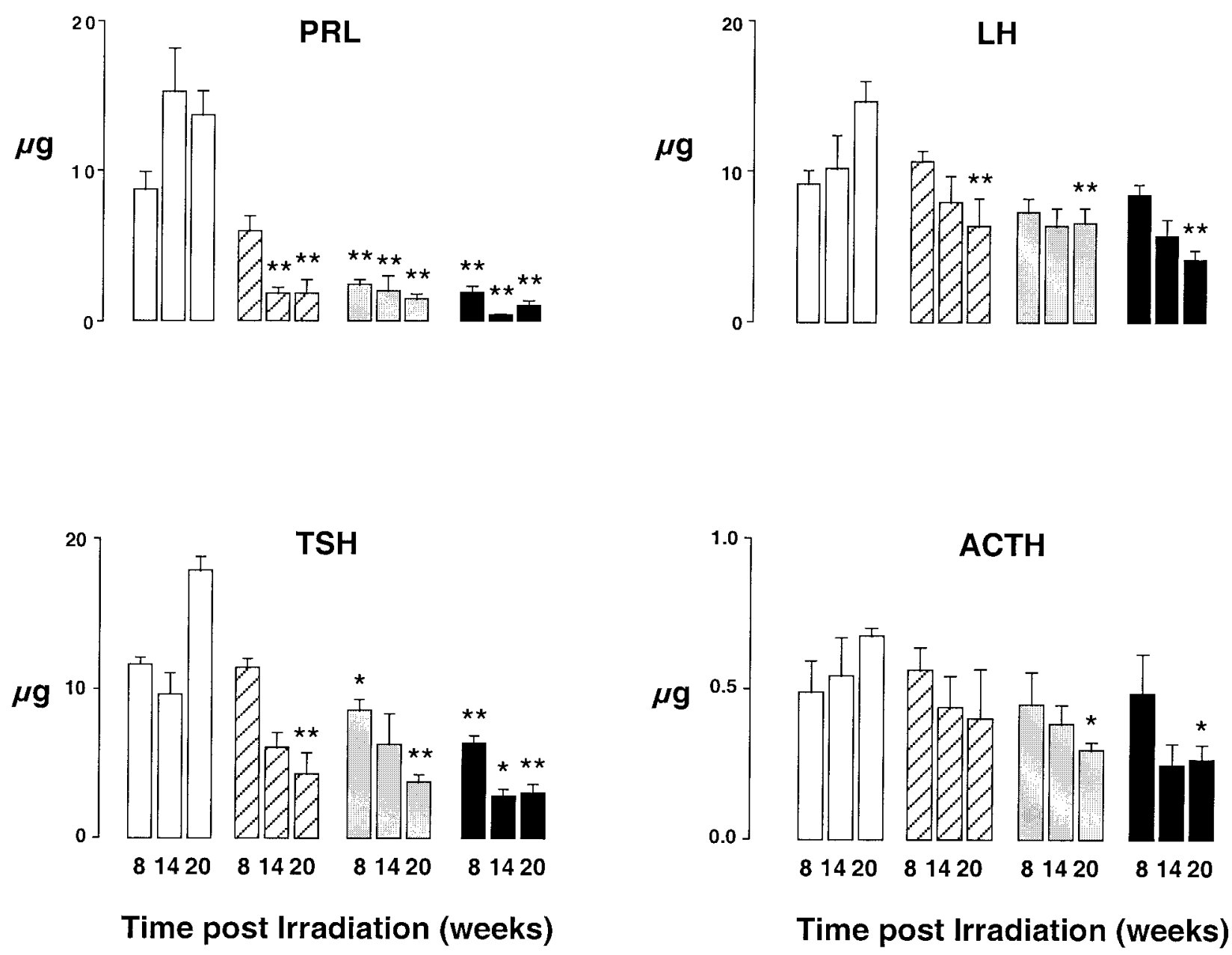

\section{Time post Irradiation (weeks)}

Figure 3 Multiple pituitary hormone deficiencies are induced by cranial irradiation in the rat. The groups are the same as in Fig. 1 and received 0 Gy (Control group, open bars), 20 Gy (hatched bars), 22 Gy (shaded bars) or 24 Gy irradiation (solid bars). PRL, TSH, LH and ACTH contents were measured by RIA in pituitaries from rats killed 8, 14 or 20 weeks after cranial irradiation, and expressed as $\mu \mathrm{g} /$ pituitary. Note the differences in scale, reflecting the differing abundance of these hormones in pituitary extracts. Data are means + S.E.M. ${ }^{*} P<0 \cdot 05 ;{ }^{* *} P<0 \cdot 01$ compared with the corresponding time point in controls (Dunnett's test).

1968). We believe that the reductions in pituitary size and individual pituitary hormone stores will reflect a reduced cell number and secretory capacity, but did not evaluate this directly. However, rats that were stunted after neonatal irradiation continued to show GH pulsatility, but with significantly lower pulse amplitude (Mosier et al. 1985). There is no similar information on other hormone profiles, but this can now be obtained in our model using microsampling methodology for conscious rats (Clark et al. 1986).

A number of conclusions may be drawn from our results. Firstly, the pituitary hormone axes in young adult rats are differentially sensitive to irradiation damage, with respect to onset, rate of progression and severity. Secondly, the GH and PRL axes are the first to be affected, and are the most sensitive to the dose of radiation. Thirdly, graded progres- sive multiple hormone deficiencies can be produced with larger doses of irradiation. These findings closely parallel the observations made after cranial irradiation in humans, but with some important differences.

The most surprising finding was that PRL contents were as sensitive as $\mathrm{GH}$ in irradiated rats, and decreased dramatically with time and dose. In the light of the radiation-induced hyperprolactinaemia reported in the human, the latter observation was unexpected. It is possible, however, that the increased serum PRL in the irradiated human, which is often transient, is itself associated with a reduction in pituitary PRL content - implying that, in the human, the overall impact on PRL synthesis and release reflects both hypothalamic and pituitary damage. Hypothalamic damage would be expected to increase PRL secretion in the rat, but the effects of 

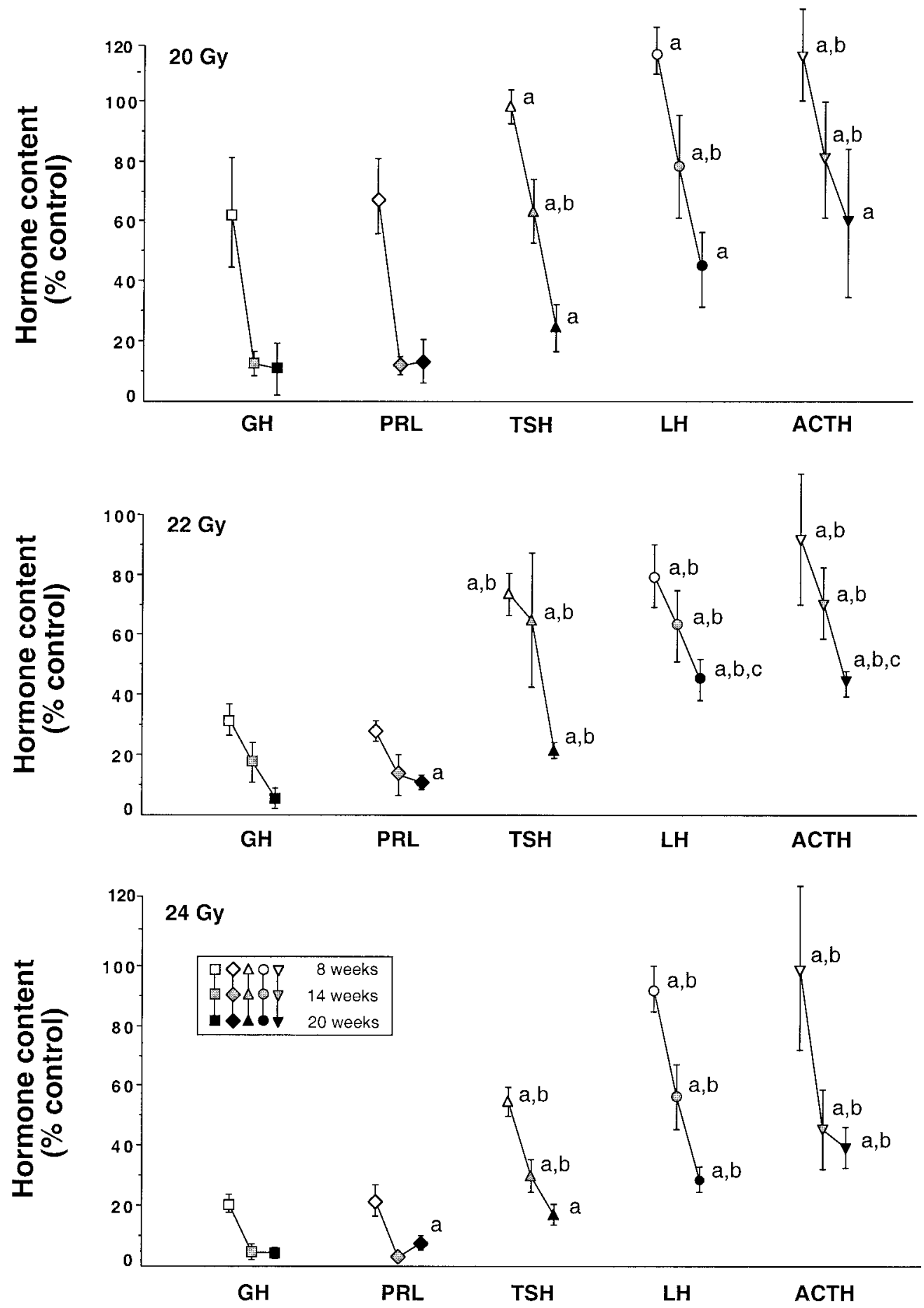

Figure 4 Differential effects of different doses of cranial irradiation on pituitary hormone deficiencies. Comparative decay curves for each hormone were constructed by expressing each hormone content as a percentage of that hormone content in the non-irradiated animals at the same time point, normalized to $100 \%$. Open symbols, 8 weeks; shaded symbols, 14 weeks; solid symbols, 20 weeks. Data are shown as means \pm S.E.M. The relative hormone contents measured at the same time point and irradiation dose were compared; significant differences $(P<0 \cdot 05$, Student-Newmann-Keuls) are indicated by letters: a, compared with $\mathrm{GH}$; b, compared with PRL; c, compared with TSH. 
irradiation on PRL are complex (Guo et al. 1997) and it is possible that the doses of irradiation used may have been sufficient to damage pituitary PRL cells directly. Increased levels of apoptosis have been found in pituitaries after 20 Gy irradiation (Guo et al. 1997), and greater doses of irradiation delivered to isolated rat pituitary GH cells compromised their survival and capacity to secrete $\mathrm{GH}$ (Hochberg et al. 1983).

Another difference between rat and human concerns the order of appearance of other hormone deficiencies. In the rat, TSH was the next most sensitive, followed by LH and ACTH, whereas in humans the order is usually $\mathrm{LH}$ followed by ACTH and TSH (Littley et al. 1989a). However, the human data derive from functional evaluation and provocative testing, whereas our rat data measured hormone contents directly, but provided no functional evaluation. This was examined in a recent study (Roth et al. 2000) in which pubertal development was accelerated by low doses (5-6 Gy) of cranial irradiation delivered to infantile female rats, but was delayed with greater doses (9-18 Gy). The authors concluded that, with early exposure, the gonadotropin-releasing hormone (GnRH)-gonadotroph axis was sensitive to low doses of cranial irradiation, but showed in the same study that greater doses of irradiation affected serum GH concentrations without affecting other pituitary hormones, thyroxine or corticosterone (Roth et al. 2000).

The endocrine consequences of external irradiation in humans are believed to reflect hypothalamic rather than anterior pituitary damage per se, and this has a major bearing on the severity and order of appearance of multiple pituitary hormone deficiencies. Among patients with pituitary adenomas treated with doses of 500 to $1500 \mathrm{~Gy}$ by the implantation of yttrium-90 directly into the pituitary gland, the combined incidence of TSH and ACTH deficiency was only $40 \%$ at 14 years (Jadresic et al. 1987), whereas the incidence was more than $90 \%$ at 10 years among patients who received external irradiation at much lower doses, ranging from 37.5 to $42.5 \mathrm{~Gy}$ (Littley et al. 1989b). The most likely explanation for this difference is that the fields for external irradiation included the hypothalamus, whereas this structure was relatively unaffected by yttrium-90 treatment.

Other circumstantial evidence also implicates the hypothalamus as an important site of damage resulting in irradiation-induced pituitary hormone deficits in humans. This includes increased basal PRL concentrations, delayed pituitary hormone responses to releasing hormones (in patients with deficiencies of gonadotrophin and TSH) and normal GH responses to GH-releasing hormone (GHRH) in patients with subnormal serum $\mathrm{GH}$ responses to arginine or insulin-induced hypoglycaemia. Reduced spontaneous secretion of GH despite normal serum GH responses to pharmacological stimuli also suggests a hypothalamic site of damage induced by cranial irradiation. If this is true, the order of appearance of individual pituitary endocrine deficits may reflect their relative dependence on hypothalamic integrity rather than any intrinsic differential sensitivity to direct irradiation at the pituitary level (though a contribution from the latter in the presence of greater doses of irradiation cannot be excluded).

Both clinical and experimental evidence points to the importance of trophic actions of hypothalamic factors for normal pituitary function. Excessive GHRH markedly increases pituitary GH cell number (Stefaneanu et al. 1989) and stimulates GH synthesis (Barinaga et al. 1985) in rodents, whereas a loss in GHRH receptor signalling causes GH cell hypoplasia and GH deficiency (Jansson et al. 1986, Godfrey et al. 1993). Parallel observations have been made in humans: pituitary GH cell hyperplasia and acromegaly result from ectopic GHRH production (Thorner et al. 1982), whereas a lack of GHRH signalling results in pituitary hypoplasia and profound GH deficiency (Maheshwari et al. 1998).

It is possible that such trophic effects could play an important role in the differential sensitivity to irradiation amongst the different hypothalamo-pituitary hormone systems. The pituitary hormone cell types may differ in the extent to which they rely on hypothalamic trophic support. Another possibility is that the different hypothalamic neuroendocrine cell groups may show intrinsic differences in their sensitivity to irradiation damage, in their proliferative or repair capacities or in their propensity to apoptose. These hypotheses can now be tested in our rodent model by combining direct measurements of hypophysiotrophic hypothalamic peptide expression and histological assessments of these neuronal cell groups after irradiation at doses eliciting different degrees of pituitary hormone deficiency.

A greater recognition of the importance of hypophysiotrophic support for pituitary function could also have a bearing on treatment for radiation-induced hypopituitarism in humans. A significant proportion of patients with radiation-induced GH deficiency and disturbances in GH pulsatility (Spoudeas et al. 1996) can release GH in response to an acute bolus of $\mathrm{GH}$ secretagogues (Ogilvy-Stuart et al. 1994); they may be candidates for chronic GHRH analogue treatment to build up their pituitary GH reserve (Ogilvy-Stuart et al. 1997, Achermann et al. 1999).

Schunior et al. (1994) used a rodent model of irradiation-induced stunting to evaluate the impact of prior methotrexate or prednisone treatments, and Winterer et al. (1988) showed that the radiosensitivity of the GnRH/gonadotroph axis in rodents could be altered by concurrent treatment with $\mathrm{GnRH}$ analogues. We hope to use our rodent model to test specific interventions with hypophysiotrophic factors that might prevent or reduce the occurrence, delay the progression, or ameliorate the severity, of long-term multiple endocrine deficits caused by cranial irradiation. 


\section{Acknowledgements}

We thank Mr D A Broadbent for expert technical assistance with the irradiations. We are also grateful for the continuing supply of reagents for pituitary hormone assays provided by NIDDK.

\section{References}

Achermann JC, Hindmarsh PC, Robinson ICAF, Matthews DR \& Brook CG 1999 The relative roles of continuous growth hormone-releasing hormone $\left(\mathrm{GHRH}(1-29) \mathrm{NH}_{2}\right)$ and intermittent somatostatin(1-14)(SS) in growth hormone $(\mathrm{GH})$ pulse generation: studies in normal and post cranial irradiated individuals. Clinical Endocrinology 51 575-585.

Barinaga M, Bilezikjian LM, Vale WW, Rosenfeld MG \& Evans RM 1985 Independent effects of growth hormone releasing factor on growth hormone release and gene transcription. Nature $\mathbf{3 1 4}$ 279-281.

Clark RG, Chambers G, Lewin J \& Robinson ICAF 1986 Automated repetitive microsampling of blood: growth hormone secretion in conscious male rats. Journal of Endocrinology 111 27-35.

Godfrey P, Rahal JO, Beamer WG, Copeland NG, Jenkins NA \& Mayo KE 1993 GHRH receptor of little mice contains a missense mutation in the extracellular domain that disrupts receptor function. Nature Genetics 4 227-232.

Guo YP, Hendry JH, Morris ID, Davis JR \& Beardwell CG 1997 Cell proliferation and death in the irradiated pituitary gland and its modification by growth stimulants. International Journal of Radiation Oncology and Biological Physics 38 175-181.

Harrop JS, Davies TJ, Capra LG \& Marks V 1976 Hypothalamicpituitary function following successful treatment of intracranial tumours. Clinical Endocrinology 5 313-321.

Hochberg Z, Kuten A, Hertz P, Tatcher M, Kedar A \& Benderly A 1983 The effect of single-dose radiation on cell survival and growth hormone secretion by rat anterior pituitary cells. Radiation Research 94 508-512.

Jadresic A, Jimenez LE \& Joplin GF 1987 Long-term effect of 90Y pituitary implantation in acromegaly. Acta Endocrinologica 115 301-306.

Jansson JO, Downs TR, Beamer WG \& Frohman LA 1986 Receptor-associated resistance to growth hormone-releasing factor in dwarf 'little' mice. Science 232 511-512.

Lam K, Tse V, Wang C, Yeung R \& Ho J 1991 Effects of cranial irradiation on hypothalamic-pituitary function - a 5 year longitudinal study in patients with nasopharyngeal carcinoma. Quarterly Journal of Medicine 78 165-176.

Littley MD, Shalet SM, Beardwell CG, Ahmed SR, Applegate G \& Sutton ML 1989a Hypopituitarism following external radiotherapy for pituitary tumours in adults. Quarterly Journal of Medicine $\mathbf{7 0}$ 145-160.

Littley MD, Shalet SM, Beardwell CG, Robinson EL \& Sutton ML $1989 b$ Radiation-induced hypopituitarism is dose-dependent. Clinical Endocrinology 31 363-373.

Maheshwari HG, Silverman BL, Dupuis J \& Baumann G 1998 Phenotype and genetic analysis of a syndrome caused by an inactivating mutation in the growth hormone-releasing hormone receptor: dwarfism of Sindh. Journal of Clinical Endocrinology and Metabolism 83 4065-4074.

Mosier HD 1988 Growth failure in the head-irradiated rat. Hormone Research 30 77-83.

Mosier HD Jr \& Jansons RA 1968 Pituitary content of somatotropin, gonadotropin, and thyrotropin in rats with stunted linear growth following head $\mathrm{x}$-irradiation. Proceedings of the Society for Experimental Biology and Medicine 128 23-26.

Mosier HD Jr \& Jansons RA 1971 Allometry of body weight and tail length after head x-irradiation in rats. Growth 35 23-31.
Mosier HD Jr, Jansons RA, Swingle KF, Sondhaus CA, Dearden LC \& Halsall LC 1985 Growth hormone secretion in the stunted head-irradiated rat. Pediatric Research 19 543-548.

Mosier HD Jr, Jansons RA, Swingle KF \& Dearden LC 1986 Dissociation of catch-up growth control and neural control of growth hormone secretion in the stunted head-irradiated rat. Pediatric Research 20 261-264.

Mosier HD, Crinella FM, Yu J, Culler FC \& Jansons RA 1993 Food efficiency in rats following brain lesions which affect target body size: implications on the set point for target size. Growth Development and Aging 57 223-231.

Ogilvy-Stuart AL, Wallace WH \& Shalet SM 1994 Radiation and neuroregulatory control of growth hormone secretion. Clinical Endocrinology 41 163-168.

Ogilvy-Stuart AL, Stirling HF, Kelnar CJ, Savage MO, Dunger DB, Buckler JM \& Shalet SM 1997 Treatment of radiation-induced growth hormone deficiency with growth hormone-releasing hormone. Clinical Endocrinology 46 571-578.

Overmier JB, Carroll ME, Patten R, Krivit W \& Kim TH 1979 Cranial irradiation of young rats impairs later learning and growth. Physiology and Behavior 23 179-184.

Richards GE, Wara WM, Grumbach MM, Kaplan SL, Sheline GE \& Conte FA 1976 Delayed onset of hypopituitarism: sequelae of therapeutic irradiation of central nervous system, eye, and middle ear tumors. Journal of Pediatrics 89 553-559.

Roth C, Schmidberger H, Schaper O, Leonhardt S, Lakomek M, Wuttke W \& Jarry H 2000 Cranial irradiation of female rats causes dose-dependent and age-dependent activation or inhibition of pubertal development. Pediatric Research 47 586-591.

Samaan NA, Vieto R, Schultz PN, Maor M, Meoz RT, Sampiere VA, Cangir A, Ried HL \& Jesse RH Jr 1982 Hypothalamic, pituitary and thyroid dysfunction after radiotherapy to the head and neck. International Journal of Radiation Oncology and Biological Physics 8 1857-1867.

Schunior A, Zengel AE, Mullenix PJ, Tarbell NJ, Howes A \& Tassinari MS 1990 An animal model to study toxicity of central nervous system therapy for childhood acute lymphoblastic leukemia: effects on growth and craniofacial proportion. Cancer Research $\mathbf{5 0}$ $6455-6460$

Schunior A, Mullenix PJ, Zengel AE, Landy H, Howes A \& Tarbell NJ 1994 Radiation effects on growth are altered in rats by prednisone and methotrexate. Pediatric Research 35 416-423.

Shalet SM \& Brennan BM 1998 Growth and growth hormone status following treatment for childhood leukaemia. Hormone Research $\mathbf{5 0}$ $1-10$.

Spoudeas HA, Hindmarsh PC, Matthews DR \& Brook CG 1996 Evolution of growth hormone neurosecretory disturbance after cranial irradiation for childhood brain tumours: a prospective study. Journal of Endocrinology 150 329-342.

Stefaneanu L, Kovacs K, Horvath E, Asa SL, Losinski NE, Billestrup N, Price J \& Vale W 1989 Adenohypophysial changes in mice transgenic for human growth hormone-releasing factor: a histological, immunocytochemical, and electron microscopic investigation. Endocrinology 125 2710-2718.

Thames HD \& Hendry JH 1987 In Fractionation in Radiotherapy, p 289. London: Taylor \& Francis Ltd.

Thorner MO, Perryman RL, Cronin MJ, Rogol AD, Draznin M, Johanson A, Vale W, Horvath E \& Kovacs K 1982 Somatotroph hyperplasia. Successful treatment of acromegaly by removal of a pancreatic islet tumor secreting a growth hormone-releasing factor. Journal of Clinical Investigation 70 965-977.

Winterer J, Barnes KM, Lichter AS, Deluca AM, Loriaux DL \& Cutler GB Jr 1988 Effect of gonadotropin secretion rate on the radiosensitivity of the rat luteinizing hormone-releasing hormone neuron and gonadotroph. Endocrinology 122 884-890.

Received in final form 5 February 2001

Accepted 8 February 2001 